CERN-TH-98-170

RI-98-6

hep-th/9805143

\title{
Extensivity Versus Holography in Anti-de Sitter Spaces
}

\author{
J.L.F. Barbón ${ }^{\mathrm{a}, \mathrm{l}}$ and E. Rabinovici ${ }^{\mathrm{b}, 2}$ \\ ${ }^{a}$ Theory Division, CERN \\ CH-1211, Geneva 23 \\ Switzerland \\ ${ }^{\mathrm{b}}$ Racah Institute of Physics \\ The Hebrew University \\ Jerusalem, 91904, Israel
}

\begin{abstract}
We study the dimensionality manifested in the $A d S / C F T$ correspondence. We show that the dimensionality as expressed by the high temperature behavior of a system has a holographic nature also at the quantum level. The emergence of the $A d S$ black hole as a master field at high temperature leads to the screening of the extra dimensions in its excluded volume.
\end{abstract}

$05 / 98$

1 barbon@mail.cern.ch, ${ }^{2}$ eliezer@vxcern.ch 


\section{Introduction}

The idea that a gravitational system may posses far less degrees of freedom than usualy expected for a field theory [1] has emerged yet again in the study of the large $N$ limit of certain conformal field theories (CFT). The blowing up of various near-horizon supergravity solutions associated to various arrangements of $N$ coincident branes of string theory and $M$-theory suggests, in a certain limit, a correspondence between the brane theories and various types of manifolds containing an Anti- de Sitter $(A d S)$ space as well as compact components and fluxes [2]. The geometry is smooth enough as long as $N$ is very large and the fixed value of $\lambda N$ is large enough. Where $\lambda$ is the string coupling. It is conjectured that such throat geometries encode in a manner a possible large $N$ master field for the associated CFT (see also [3] for early suggestions in this regard).

The simplest examples of the brane configurations involve $\mathcal{N}=4$ Super Yang-Mills (SYM) in $d=4$, from the D3-brane solution of IIB string theory, the six-dimensional $(2,0)$ CFT from the $M 5$-solution of eleven-dimensional supergravity, as well as the threedimensional CFT with $S O(8)$ symmetry coming from the $M 2$-solution, but other examples with various dimensions, gauge groups and supersymmetries can be found in the literature. In all known examples, both the $A d S$ and compact manifolds have constant radius of curvature, which we denote by $b$. It is related to the Planck length in terms of the number $N$ of branes used to define the CFT by:

$$
b=c \ell_{P} N^{\delta}
$$

with $\delta$ positive: $\delta=1 / 4, c=1$ for $\mathcal{N}=4 \mathrm{SYM}, \delta=1 / 3, c=2 \pi^{1 / 3}$ for the $(2,0)$ theory, and $\delta=1 / 6, c=(\pi / \sqrt{2})^{1 / 3}$ for the $M 2$ theory. The condition for a low energy description of the geometry to be smooth involves $b \gg \ell_{P}$, with $\ell_{P}$ the $D$-dimensional Planck length. That leads to the large $N$ limit $N \gg 1$ of the CFT.

An algorithm for computing CFT observables in the supergravity framework was developed in [4] and [5] (see [6] for earlier work). Roughly speaking, the classical supergravity action, as a function of boundary data at infinity, is a generating functional for correlators of the CFT in the planar $N=\infty$ limit.

Given that the supergravity or string theory of the near-horizon throat is eleven or ten dimensional, this can be regarded as a rather explicit realization of the holographic idea [1]: some of the dimensions are confined. 
In this note we discuss the mechanism by which the full ten or eleven dimensional physics of the supegravity side would not be liberated and exposed in certain extreme regimes. In particular, a physical characterization of the number of effective dimensions in quantum field theory, is provided by the high-temperature asymptotics of the free energy. For a field theory in $d$ space-time dimensions, the large temperature asymptotics of the free energy density is

$$
\frac{F}{T V} \rightarrow-n_{*} T^{d-1}
$$

where $n_{*}$ is the effective number of degrees of freedom at high temperature. This estimate is good as long as $V T^{d-1} \gg 1$, so that finite-size effects are negligible, and $M \ll T \ll \Lambda_{U V}$, where $M$ is any characteristic scale of the theory, and $\Lambda_{U V}$ is the ultraviolet cut-off in the case of an effective theory. A nice feature of (1.2) is its independence on the detailed short distance structure of the theory, which affects only the vacuum energy, subtracted from (1.2).

According to the CFT/AdS mapping, the free energy (1.2) would be computable in a $1 / N$ expansion, in terms of the thermal free energy in the supergravity background. In principle, at sufficiently high temperatures, but still small compared to the Planck mass, one might be able to uncover the full ten or eleven dimensionality of the supergravity background. In Section 2 we discuss and review how this question is resolved in a rather non-trivial way, to leading order. This occurs via an important refinement of the CFT/AdS mapping, proposed in [5]. The refinement consists of allowing phase transitions in the gravitational background, in particular gravitational collapse. To be more explicit, the partition function of the boundary $d$-dimensional conformal theory is instructed to read

$$
Z_{C F T}=\sum_{i} e^{-I\left(X_{i}\right)}
$$

where $X_{i}$ are the various manifolds whose boundary $\partial X_{i}$ is the space-time manifold. some of them will have holographic features. They have the form $X=E_{d+1} \times M_{D-d-1}$ with $E_{d+1}$ an Einstein manifold of negative curvature and $M_{D-d-1}$ a compact manifold which, in the simplest cases of SCFT with 16 supercharges, is a sphere $\mathbf{S}^{D-d-1}$, threaded by a flux of the associated brane field. Both factors, $E_{d+1}$ and $M_{D-d-1}$, have comparable constant radius of curvature, $b$. It is this property of the manifold $M_{D-d-1}$ rather than its detailed topology which counts for the high tempreture considerations studied in this note. The quantity $I(X)$ denotes the full effective action of the supergravity or string theory on $X$. 
Using known results from [7], one can see that thermal $A d S$ space is unstable against the formation of an $A d S$ black hole at sufficiently high temperatures, and the classical entropy of the $A d S$ black hole ensures the correct scaling of the $N=\infty$ entropy of the CFT. Quarks are deconfined at high temperature, the extra dimensions are not.

However the possibility of exposing the full $D$ dimensions of the supergravity manifold $X$ reappears at the level of the one-loop thermal free energy, that is while calculating the $O\left(N^{0}\right)$ free energy of the CFT from the supergravity side. That calculation amounts to tracing over the perturbative excitations of supergravity in $X$. In a regular field theory such a quantum calculation reveals the full space time dimensions of the manifold on which the excitations propagate. In Section 3 we show that that would be the case also for the vacuum $A d S$ space but not for the black hole in $A d S$ space. As determined already at the classical level, it is the black hole configuration which dominates at high temperature, allowing for the extra dimensions to be "confined" at all temperatures. We isolate that piece of the $X$ manifolds which can lead to this form of holography and find it behind the horizon of the black hole. We conclude by indicating that the gauge systems at strong coupling are censored from showing the Hagedorn transition, and we also speculate on the string theory nature of the phase transition occuring in the supergravity picture, and that holography is probably violated in any case for Dp-branes with $p \geq 5$ 目.

\section{Classical Sugra, or Planar CFT}

In this section, we review the realization of holography in the classical, or planar approximation, as treated in [5] and [9].

At finite temperature, when the space manifold of the CFT is a sphere, $\partial X=$ $S_{\beta}^{1} \times \mathbf{S}^{d-1}$. There are two possible choices for the $D$-dimensional manifold, according to [5]: $X_{1}=\frac{A d S_{d+1}^{+}}{\mathrm{Z}} \times M_{D-d-1}$ and $X_{2}=A d S_{\mathrm{bh}} \times M_{D-d-1}$, i.e. a periodically identified $A d S_{d+1}$ or an $A d S_{d+1}$ black hole times the compact space $M_{D-d-1}$ which, for the simplest maximally supersymmetric cases, is a sphere $\mathbf{S}^{D-d-1}$. The manifold $X_{1}$ is given by the metric

$$
d s^{2}\left(X_{1}\right)=\left(1+r^{2} / b^{2}\right) d t^{2}+\frac{d r^{2}}{1+r^{2} / b^{2}}+r^{2} d \Omega_{d-1}^{2}+b^{2} d s^{2}(M)
$$

2 While looking at a Paris mirror, we found [8], where some of the issues dealt with in this paper, are also discussed. 
with topology $S^{1} \times \mathbf{R}^{d} \times M_{D-d-1}$. The time coordinate is identified with period $t \equiv t+\beta_{0}$. The proper inverse temperature in the asymptotic, large $r$, limit is $\beta(r) \rightarrow \beta_{0} r / b$. The radius of the asymptotic $\mathbf{S}^{d-1}$ at the boundary goes like $R(r) \rightarrow r$, so that the ratio of spatial size and inverse temperature (the only relevant parameter for a CFT) is $R / \beta \rightarrow$ $b / \beta_{0}$.

The manifold $X_{2}$ has the same large $r$ asymptotics but a different global topology, $\mathbf{R}^{2} \times \mathbf{S}^{d-1} \times M_{D-d-1}$. The metric represents an $A d S$ black hole with an outer horizon at $r_{0}$ :

$$
d s^{2}\left(X_{2}\right)=\mu(r) d t^{2}+\frac{d r^{2}}{\mu(r)}+r^{2} d \Omega_{d-1}^{2}+b^{2} d s^{2}(M)
$$

where

$$
g_{t t}=\mu(r)=\frac{r^{2}}{b^{2}}+1-\frac{r_{0}^{d-2}}{r^{d-2}}\left(\frac{r_{0}^{2}}{b^{2}}+1\right) .
$$

Regularity at the horizon fixes the period of $t$ to be

$$
\beta_{0}=\frac{4 \pi^{2} b^{2} r_{0}}{r_{0}^{2} d+(d-2) b^{2}} .
$$

The maximum at $r_{0}=b \sqrt{1-2 / d}$ marks the smallest black holes that can contribute to the CFT thermodynamics. The minimum temperature required for it to exist is $\beta_{\max }(d)=$ $2 \pi^{2} b / \sqrt{d(d-2)}$. In the rest of the paper, we will restrict ourselves to the case $d>2$, where the previous formulas make sense.

The classical action

$$
I_{c \ell}=-\frac{1}{16 \pi G_{d+1}} \int_{X}(R+\Lambda),
$$

when evaluated on any of the manifolds $X_{i}$ gives3, using (1.1):

$$
\begin{aligned}
I(X)_{c \ell} & =\frac{d}{8 \pi G_{d+1}} \frac{\operatorname{Vol}\left(E_{d+1}\right)}{b^{2}} \\
& =N^{\delta(D-2)} \frac{2 c^{D-2}}{(2 \pi)^{D-3}}\left|\Omega_{d-1}\right|\left|\Omega_{M}\right| \frac{\beta_{0}}{b^{d+1}}\left(r_{\max }^{d}-r_{\min }^{d}\right),
\end{aligned}
$$

where $\left|\Omega_{d-1}\right|$ denotes the volume of the $\mathbf{S}^{d-1}$ sphere, and $\left|\Omega_{M}\right|=b^{1+d-D} \operatorname{Vol}(M)$ stands for the normalized volume of $M_{D-d-1}$. When $M$ is a sphere, we have $\left|\Omega_{M}\right|=\operatorname{Vol}\left(\mathbf{S}^{D-d-1}\right)$.

For $\beta_{0}>\beta_{\max }(d)$, only the manifold $X_{1}$ exists, and thus its features will determine the free energy and the entropy at these low temperatures. Classically, there is no obstruction

3 In our conventions $16 \pi G_{D}=(2 \pi)^{D-3} \ell_{P}^{D-2}$. 
to locate the mininum radius of $X_{1}$ at $r_{\min }=0$. As a result, the classical effective action of $X_{1}$ is proportional to $\beta_{0}$, and therefore it is to be interpreted as a vacuum energy, which does not contribute to the entropy. We conclude from here that the entropy in the low temperature phase is subleading in the large $N$ expansion.

At higher temperatures we must compare the actions of $X_{1}$ and $X_{2}$. The dominant manifold in the 'planar' limit is obtained by comparing the volumes of $X_{1}$ and $X_{2}$, regularized at a large radial distance $r_{\max }$. In evaluating the volume of $X_{2}$, one must account for the fact that the boundary temperatures induced by $X_{1}$ and $X_{2}$ are slightly different. For large $R$, one obtains

$$
\beta_{0}^{\prime} R^{d}=\beta_{0} R^{d}+\frac{1}{2} \beta_{0} b^{2} r_{0}^{d-2}\left(1+r_{0}^{2} / b^{2}\right)+O\left(R^{-d}\right),
$$

where $\beta_{0}$ is the period of $t$ in $X_{1}$ and $\beta_{0}^{\prime}$ the period of $t$ in $X_{2}$. The "vacuum" contribution of $X_{2}$ is non trivial, and one finds

$$
I\left(X_{2}\right)_{c \ell}=I\left(X_{1}\right)_{c \ell}+N^{\delta(D-2)} \frac{c^{D-2}}{(2 \pi)^{D-3}}\left|\Omega_{d-1}\right|\left|\Omega_{M}\right| b^{-d-1} \beta_{0}\left(b^{2} r_{0}^{d-2}-r_{0}^{d}\right),
$$

leading to $X_{1}$ domination when $r_{0}<b$ (thus, there is actually a window where $X_{2}$ exists but does not dominate), and $X_{2}$ domination when $r_{0}>b$. In particular, $X_{2}$ dominates at high temperatures, where $r_{0} \sim b^{2} / \beta_{0}$, and $\beta_{0} \ll b$. The resulting large $N$ free energy at high temperatures is given by

$$
\beta F(\beta)_{\text {planar }}=I\left(X_{2}\right)_{c \ell}-I\left(X_{1}\right)_{c \ell}=-N^{\delta(D-2)} C_{d}\left(\frac{R}{\beta}\right)^{d-1},
$$

where the $d$-dependent constant $C_{d}$ is given by

$$
C_{d}=\left(\frac{2 \pi}{d}\right)^{d} \cdot \frac{c^{D-2}}{(2 \pi)^{D-d-3}} \cdot\left|\Omega_{d-1}\right|\left|\Omega_{M}\right| .
$$

The corresponding energy is

$$
E\left(X_{2}\right)_{\text {planar }}=\beta E_{\mathrm{vac}}+N^{\delta(D-2)}(d-1) C_{d} R^{d-1} \beta^{-d},
$$

and the entropy

$$
S\left(X_{2}\right)_{\text {planar }}=+N^{\delta(D-2)} d \cdot C_{d}\left(\frac{R}{\beta}\right)^{d-1}
$$

or, in microcanonical form

$$
S\left(X_{2}\right)_{\text {planar }}=N^{\delta(D-2) / d} \cdot d(d-1)^{\frac{1-d}{d}} \cdot\left(C_{d}\right)^{\frac{1}{d}}\left[\left(E-E_{\mathrm{vac}}\right) R\right]^{\frac{d-1}{d}} .
$$

It is important to realize that this formula applies with the subtracted energy only, by the way in which it was derived via the canonical entropy. In the following, unless otherwise stated, we shall always refer to the canonical entropy, expressed as a function of the temperature which is the one with a transparent large $N$ expansion, due to the fact that the temperature is held fixed in the large $N$ limit. 


\section{One-loop Sugra, or $1 / N$ CFT corrections}

In this section we estimate the $O\left(N^{0}\right)$ correction to the CFT entropy, in the high temperature limit. As stated in the introduction, this involves computing the one-loop free energy of the supergravity or string theory based on the D-dimensional manifold $X$ :

$$
\beta F(\beta, X)_{\text {toroidal }}=I(X)_{1-\text { loop }}=\frac{1}{2} \operatorname{Tr} \log \left(I^{\prime \prime}\right)_{X},
$$

with $\left(I^{\prime \prime}\right)_{X}$ the complete fluctuation kernel of the theory around the manifold $X$, including all fields and gauge fixing terms (in the full string theory, it is the torus free energy). It is this contribution which could be suspect of probing the full $D$ dimensions at sufficiently high temperature.

Notice that this free energy has a multiplicity given by the number of species in the supergravity background, but one of the successes of the proposal in [2] is precisely the fact that such species multiplicity is of order $O(1)$ in the large $N$ limit. In other words, all explicit dependence in $N$ is contained in the geometry of $X$ on the supergravity side.

The thermal entropy we would derive from (3.1) has a standard quantum mechanical interpretation in the supergravity side, coming from tracing over physical states propagating in the supergravity background. This is in sharp contrast with the planar entropy computed in the previous section, which was classical or "topological" on the supergravity side. In fact, the very ability to regard the "intrinsic" topological entropy of Hawking and Gibbons [10], as a planar approximation to the standard thermal entropy of a say, gauge theory, is what is meant by a "microscopic explanation" of the Beckenstein-Hawking entropy.

For sufficiently high temperatures, we can use the WKB approximation to estimate the one-loop partition function (3.1) for the supergravity case, as the integral over the space-time manifold of the free energy density of the thermal gas. The free energy density is approximated by the red-shifted flat energy density [11]:

$$
\beta F(\beta)_{W K B}=\int d^{D} x \sqrt{g} f\left(x, \beta_{\mathrm{loc}}\right),
$$

with the local red-shifted inverse temperature being $\beta_{\text {loc }}=\beta \sqrt{g_{00}}$. The flat space free energy density for a field of mass $m$ is

$$
f(\beta, m)_{D}=-n_{\text {dof }} \frac{\Gamma(D / 2)}{\Gamma(D) \pi^{D / 2}} \beta^{-D} \int_{\beta m}^{\infty} d z \frac{\left(z^{2}-\beta^{2} m^{2}\right)^{\frac{D-1}{2}}}{e^{z}-(-1)^{F}} .
$$


The mass $m$ could represent a global gap in the spectrum of the theory, for the excited string states $m \sim 1 / \sqrt{\alpha^{\prime}}$ and for excited Kaluza-Klein states $m \sim 1 / R_{K K}$. We denote by $n_{\text {dof }}$ the number of degrees of freedom of the field.

The large mass asymptotics of the flat space free energy is of the form:

$$
f(\beta, m)_{D} \sim-\beta^{-D}(\beta m)^{\frac{D-1}{2}} e^{-\beta m}+O\left(e^{-2 \beta m}\right)
$$

Therefore, in the case of Kaluza-Klein thresholds $m_{\text {loc }} \sim 1 / R_{\text {loc }}$, whenever $\beta_{\text {loc }} m_{\text {loc }}>1$, the effective dimensionality over which the free energy is integrated drops by the number of compact dimensions, up to the exponentially suppresed corrections above, of order $e^{-\beta_{\text {loc }} / R_{\text {loc }}}$. What remains is the massless free energy now integrated over the dimensionally reduced space.

$$
\begin{aligned}
\beta F(\beta)_{W K B} & =-n_{*}\left(d_{\mathrm{eff}}\right) a\left(d_{\mathrm{eff}}\right) \int d^{d_{\mathrm{eff}}} x \sqrt{g}\left(\beta \sqrt{g_{00}}\right)^{-d_{\mathrm{eff}}} \\
& =-n_{*}\left(d_{\mathrm{eff}}\right) a\left(d_{\mathrm{eff}}\right) \beta^{-d_{\mathrm{eff}}} \widetilde{\operatorname{Vol}}\left(X_{\mathrm{eff}}\right),
\end{aligned}
$$

where $d_{\text {eff }}$ is the effective dimension. The constant $a(d)=\Gamma(d / 2) \zeta(d) \pi^{-d / 2}$, and $n_{*}$ is the effective number of degrees of freedom at high temperature. For $n_{B}\left(n_{F}\right)$ bosonic (fermionic) degrees of freedom, it is given by

$$
n_{*}(d)=n_{B}+\left(1-\frac{1}{2^{d-1}}\right) n_{F} .
$$

We find it convenient to write the answer in terms of $\widetilde{\operatorname{Vol}}\left(X_{\text {eff }}\right)$, the volume of $X_{\text {eff }}$ in the so-called optical metric (see for example [1])

$$
\widetilde{g}_{\mu \nu}=\frac{g_{\mu \nu}}{g_{00}} .
$$

If we have several regions with different number of effective dimensions (depending on the local value of the temperature), the general WKB expression of the free energy, up to the large mass corrections above, and cross-over effects, is given by

$$
\beta F(\beta)_{W K B} \simeq-\sum_{X_{d_{i}}} n_{*}\left(d_{i}\right) a\left(d_{i}\right) \beta^{-d_{i}} \widetilde{\operatorname{Vol}}\left(X_{d_{i}}\right)
$$

There are two important comments regarding these estimates. The first is that the flat space vacuum energy has been subtracted in the expression for the flat free energy 
density (3.3) (that is why it comes out negative). This procedure is ambiguous in curved space, because it is not clear how one should red-shift the vacuum energy. In principle, each regularization prescription for the determinant, like the zeta function regularization for example, could have a different subtraction procedure for the vacuum energy, i.e. renormalization of the cosmological constant. In the following we will just proceed with these red-shift WKB estimates keeping in mind that only the free energy up to a term of the form $\beta \Lambda_{\mathrm{vac}}$ is being computed. Fortunately, such terms, linearly dependent on $\beta$, are irrelevant for the calculation of the entropy, as well as for determining perturbatively which of the manifolds $X_{i}$ dominates.

The second comment is that red-shift estimates fail near horizons, as the local temperature diverges there. This divergence can be cut-off by means of a sort of "brick-wall", as in [12]. The divergent term can be absorbed into a renormalization of the Newton constant [13]. For the purposes of our order of magnitude estimates, we will assume that a covariant regularization procedure can be used, such as zeta function regularization of the determinant in (3.1), and we determine the ultraviolet-finite part by dimensional arguments.

\subsection{One-loop in $X_{1}$}

In order to estimate the $W K B$ free energy along the lines of the previous section, we consider the optical metric of the $X_{1}$, or vacuum $A d S$ space:

$$
\widetilde{d}^{2}\left(X_{1}\right)=d t^{2}+\frac{d r^{2}}{\left(1+r^{2} / b^{2}\right)^{2}}+\frac{r^{2}}{\left(1+r^{2} / b^{2}\right)} d \Omega_{d-1}^{2}+\frac{b^{2}}{\left(1+r^{2} / b^{2}\right)} d s^{2}(M) \text {. }
$$

The optical volume of the full $X_{1}$ between $r_{\min }$ and $r_{\max }$ is

$$
\left[\widetilde{\operatorname{Vol}}\left(X_{1}\right)\right]_{z_{\min }}^{z_{\max }}=\left|\Omega_{d-1}\right|\left|\Omega_{M}\right| \beta_{0} b^{D-1} \int_{z_{\min }}^{z_{\max }} \frac{d z z^{d-1}}{\left(1+z^{2}\right)^{\frac{D}{2}}}
$$

with $z=r / b$, and the optical volume of the $A d S_{d+1}$ piece is

$$
\left[\widetilde{\operatorname{Vol}}\left(A d S_{d+1}\right)\right]_{z_{\min }}^{z_{\max }}=\left|\Omega_{d-1}\right| \beta_{0} b^{d} \int_{z_{\min }}^{z_{\max }} \frac{d z z^{d-1}}{\left(1+z^{2}\right)^{\frac{d+1}{2}}}
$$

The local temperature is larger than the Kaluza-Klein threshold of the $M_{D-d-1}$ when

$$
\left(1+\frac{r^{2}}{b^{2}}\right) \beta_{0}^{2}<b^{2}
$$


At high temperatures, this is equivalent to $r<b^{2} / \beta_{0}$. This means that, for $r>b^{2} / \beta_{0}$ the effective dimensionality drops and we lose (do not excite) the compact $M_{D-d-1}$ factor. From (3.7):

$$
\begin{aligned}
I\left(X_{1}\right)_{1-\text { loop }} \simeq & -n_{*}(D) a(D) \beta_{0}^{-D}\left[\widetilde{\operatorname{Vol}}\left(X_{1}\right)\right]_{z=0}^{z=b / \beta_{0}} \\
& -n_{*}(d+1) a(d+1) \beta_{0}^{-d-1}\left[\widetilde{\operatorname{Vol}}\left(A d S_{d+1}\right)\right]_{z=b / \beta_{0}}^{z=\infty} \sim \\
\sim & -\left(\frac{b}{\beta_{0}}\right)^{D-1}-C\left(\frac{b}{\beta_{0}}\right)^{d-1} \sim-\left(\frac{R}{\beta}\right)^{D-1} .
\end{aligned}
$$

A clear violation of holography!?. The high-temperature asymptotics is $D$-dimensional instead of $d$-dimensional. The total high temperature entropy that one would derive from this free energy takes the form

$$
S\left(X_{1}\right)_{\text {toroidal }} \sim\left(\frac{R}{\beta}\right)^{D-1} \sim\left[\left(E-E_{\mathrm{vac}}\right) R\right]^{\frac{D-1}{D}},
$$

where the large $N$ expansion of $E-E_{\text {vac }}$ would start without a term proportional to $N^{\delta(D-2)}$. The same form of the entropy was found in [14], by algebraic considerations (perhaps subject to finite-size corrections).

On a closer examination of (3.12), we see that the asymptotic region, contributing the optical volume of $A d S_{d+1}$, scales holographically. The optical volume is finite at large $r$, and thus Anti-de Sitter space is not extensive asymptotically. The reason why neither the Kaluza-Klein modes of $M_{D-d-1}$, nor the $A d S$ modes at large radius are excited at high CFT temperatures, is the arbitrarily large redshift of the local temperature at large $r$.

The non-holographic contribution comes here from the "inside" region: $0<r<b^{2} / \beta_{0}$. In a pictorial way, inherited from the brane system that has been blown up, we can say that holography is guaranteed "up the throat", in what is interpreted as the ultraviolet region of the CFT. On the other hand, Anti-de Sitter is not holographic "down the throat", towards the infrared of the gauge theory.

\subsection{One-loop in $X_{2}$}

In estimating the one-loop free energy in the $A d S$ black hole or $X_{2}$ manifold, we split it into two contributions:

$$
I\left(X_{2}\right)_{1-\text { loop }} \simeq I\left(X_{2}\right)_{\text {horizon }}+I\left(X_{2}\right)_{\text {asymptotic }}
$$


First, we estimate the contribution from the asymptotic $A d S$ region. The radial threshold for cutting off the Kaluza-Klein modes of $M_{D-d-1}$ is, according to (3.11), $r_{\text {cut }} \sim$ $b^{2} / \beta_{0}$, but this is precisely of the order of the horizon radius $r_{\text {cut }} \sim r_{0}$ at high temperatures. The calculation is entirely analogous to that of $X_{1}$, except for the crucial fact that the "inside region" responsible for the extensive behaviour, is now missing from $X_{2}$, cut-off by the presence of the black hole horizon. Therefore, we estimate the free energy with just the optical volume of $A d S_{d+1}$

$$
\begin{aligned}
I\left(X_{2}\right)_{\text {asymptotic }} & \simeq-n_{*}(d+1) a(d+1) \beta_{0}^{-d-1}\left[\widetilde{\operatorname{Vol}}\left(A d S_{d+1}\right)\right]_{z=b / \beta_{0}}^{z=\infty} \\
& \sim-\left(\frac{b}{\beta_{0}}\right)^{d-1} \sim-\left(\frac{R}{\beta}\right)^{d-1} .
\end{aligned}
$$

A fully holographic result.

On the other hand, the first term in (3.14), $I_{\text {horizon, }}$ comes from the cap or nearhorizon region of the euclidean $X_{2}$ manifold, i.e. we slice out the region $r_{0}<r<\alpha r_{0}$, with $\alpha>1$ and of order one, chosen such that $r>\alpha r_{0}$ can be regarded as asymptotically AdS with a good accuracy. The resulting manifold $X_{\text {cap }}$ has topology Disc $\alpha \times \mathbf{S}_{\mathrm{Hor}}^{d-1} \times M_{D-d-1}$. Notice that this is not a cylindrical manifold, and therefore the one-loop effective action on $X_{\text {cap }}$ is just a vacuum energy in this space. We estimate its size as follows.

Consider the black hole metric in the $r, t$ coordinates

$$
d s_{(r, t)}^{2}=\lambda(r) d t^{2}+\frac{d r^{2}}{\mu(r)} .
$$

At a regular horizon $r=r_{0}$ we expand $\lambda(r) \sim \lambda_{0}^{\prime}\left(r-r_{0}\right)$ and $\mu(r) \sim \mu_{0}^{\prime}\left(r-r_{0}\right)$. Then, defining the coordinate $\rho=2 \sqrt{\left(r-r_{0}\right) / \mu_{0}^{\prime}}$, and the angular variable $\theta=t \sqrt{\lambda_{0}^{\prime} \mu_{0}^{\prime}} / 2$, we have a copy of $\mathbf{R}^{2}$

$$
d s^{2}=d \rho^{2}+\rho^{2} d \theta^{2} .
$$

So, locally, the metric is of the form (flat Disc) $\times$ (round sphere), the round sphere being the angles at the horizon. For the $X_{2}$ manifold in our case $\lambda(r)=\mu(r)$ and $\lambda_{0}^{\prime}=r_{0} d / b^{2}+(d-$ $2) / r_{0}$. In the regime of high temperature, we have $r_{0} \sim b^{2} / \beta_{0}$ and $\lambda_{0}^{\prime} \sim 1 / \beta_{0}$. The radius of the horizon disc, $r=\alpha r_{0}$, translates into the $\rho$ coordinate radius $\rho_{\alpha}=2 \sqrt{(\alpha-1) r_{0} / \lambda_{0}^{\prime}}$ and it extends over an area

$$
\rho_{\alpha}^{2} \sim b^{2}
$$


Since the size of the horizon sphere $\mathbf{S}_{\text {Hor }}^{d-1}$, given by $r_{0} \sim b^{2} / \beta_{0}$, is much larger than the size of the horizon disc or the compact $M_{D-d-1}$ manifold, the ultraviolet-finite part of the vacuum energy in $X_{\text {cap }}$ can be estimated on dimensional grounds as

$$
I\left(X_{2}\right)_{\text {horizon }} \sim \frac{A_{H}}{b^{d-1}} \sim\left(\frac{b^{2}}{\beta_{0}}\right)^{d-1} \frac{1}{b^{d-1}} \sim\left(\frac{R}{\beta}\right)^{d-1}
$$

The correct dimensional dependence for a massless free energy in $d$ dimensions.

\subsection{Short distance effects}

Ultraviolet divergences in the computation of (3.17) can be absorbed into a tower of higher-dimensional counterterms, present at the cut-off scale since supergravity is an effective low-energy theory. These higher derivative corrections to the low-energy effective supergravity are understood in the case of a Type IIB string theory, $D=10$, as the effect of having integrated out the massive string states.

We will show that all the short-distance effects treated in terms of a low-energy expansion, give contributions to the free energy proportional to the volume of $E_{d+1}$. Therefore, following the arguments of the section 2 we find again that the high temperature asymptotics is $d$-dimensional, proportional to $\beta^{1-d}$.

The required linear dependence with the volume of $E_{d+1}$ follows from the isometries of $X$, as each derivative of the metric brings one power of $1 / b$. In the general case, the contribution of an effective operator with $2 n$ derivatives of the metric $\mathcal{O}_{2 n} \sim \partial^{2 n}(g) \sim R^{n}$ is, when evaluated on the manifold $X$ :

$$
\left\langle\mathcal{O}_{2 n}\right\rangle_{X} \sim \int_{X} \frac{\mathcal{O}_{2 n}(X)}{\ell_{P}^{D-2 n}} \sim \frac{\operatorname{Vol}(X)}{b^{2 n} \ell_{P}^{D-2 n}} \sim N^{\delta(D-2)} \frac{\operatorname{Vol}\left(E_{d+1}\right)}{b^{d+1}} \cdot\left(\frac{1}{N^{2 \delta}}\right)^{n-1}
$$

An important restriction is that, at least when the parent theory is $D=11$ supergravity or $D=10$ superstrings, the most relevant operators have dimension two (i.e. the Ricci scalar in the Einstein-Hilbert action). In particular, there is no $D$-dimensional cosmological constant. This follows from the fact that particular examples of $X$ are scaled from the near horizon or throat region of supergravity solutions of branes, which indeed have an asymptotic region approaching $D$-dimensional Minkowski space. Therefore, in applications, the effective action is the one determined by the vacuum of the brane at asymptotic infinity, and the leading operator is the Ricci tensor and others related by the 
$D$-dimensional equations of motion, i.e. we have not added a $D$-dimensional Planck-sized cosmological constant to (2.5) (there is only one of order $\left(G_{d+1} \cdot b^{2}\right)^{-1}$. This justifies the notation in (3.18) and implies that the leading large $N$ asymptotics of the effective action goes like $N^{\delta(D-2)}$, while $1 / N^{2 \delta}$ is the expansion parameter. In general, this does not correspond to a 't Hooft-like expansion. For example, the six-dimensional $(2,0)$ theory would have a $1 / N^{2 / 3}$ expansion, with a leading "planar" term of order $N^{3}$, unlike the 't Hooft expansion of a gauge theory, with a planar leading order $\sim N^{2}$ and an expansion parameter $1 / N^{2}$.

A 't Hooft-like expansion arises when the supergravity description derives from weakly coupled string theory. In this case $D=10$ and the Planck scale is related to the string scale $\sqrt{\alpha^{\prime}}$ via the dimensionless string coupling constant $\ell_{P}=\lambda^{1 / 4} \sqrt{\alpha^{\prime}}$, with $\lambda=e^{\left.\phi_{\infty} \text {. } ⿴ 囗 ⿱ 一 一\right) ~}$ Now the low energy or $\alpha^{\prime}$ expansion is in powers of $\alpha^{\prime} / b^{2}$, and the loop expansion in powers of $\lambda$. An operator with $2 n$ derivatives as before, generated at $g$ loop order leads to

$$
\left\langle\mathcal{O}_{(2 n, g)}\right\rangle_{X} \sim \int_{X} \lambda^{2 g-2} \frac{\mathcal{O}_{2 n}(X)}{\left(\alpha^{\prime}\right)^{5-n}} \sim N^{2}\left(\frac{(\lambda N)^{2}}{N^{2}}\right)^{g}\left(\frac{1}{\sqrt{\lambda N}}\right)^{n-1} \frac{\operatorname{Vol}\left(E_{d+1}\right)}{b^{d+1}}
$$

In the $\mathcal{N}=4$ SYM theory, the 't Hooft limit holds $g_{Y M}^{2} N \sim \lambda N$ fixed, where $g_{Y M}^{2} \sim \lambda$ is the Yang-Mills coupling. Therefore, the loop expansion of the IIB theory leads to the $1 / N$ expansion in the gauge theory, while the $\alpha^{\prime}$ expansion corresponds to a strongcoupling expansion of the gauge theory in powers of the inverse large $N$ 't Hooft coupling $1 / \sqrt{g_{Y M}^{2} N} \sim 1 / \sqrt{\lambda N} \sim \alpha^{\prime} / b^{2}$. D-instanton effects on the type IIB side scale like YangMills instantons on the CFT side, and are exponentially suppresed like $e^{-N}$ in the large $N$ limit [15].

A very interesting issue is whether string perturbation theory (i.e. the 't Hooft expansion of the CFT) could break down due to light states with support on the throat geometry. Obvious candidates for this phenomenon are the thermal winding modes which could signify a Hagedorn transition down the throat, when the local temperature reaches the string scale. This condition defines a "Hagedorn radius" $r_{h}$ such that

$$
\beta_{0} \sqrt{1+r_{h}^{2} / b^{2}} \sim \sqrt{\alpha^{\prime}}
$$

4 Notice that the vacuum expectation value of the dilaton is read at asymptotic infinity in the Minkowski region. This is another manifestation of the rule stated before, that the effective action is specified with respect to the asymptotically Minkowski vacuum far away from the brane. 
In terms of the physical temperature at the boundary, we see that we need extremely high temperatures $\beta \ll R /(\lambda N)^{1 / 4}$ in order to have important Hagedorn effects on a sizeable portion of $X_{1}$. One can check [16] that such effects never upset the dominance of the smooth $X_{2}$ manifold in the large temperature phase. In fact, as long as the bulk curvature is small, i.e. $\lambda N \gg 1$, there is a thermodynamic "censorship" of Hagedorn effects. Such censorship ceases to be effective at weak 't Hooft coupling $\lambda N<1$. In this regime, however, the curvature is of order the string scale, and the whole holographic manifold $X$ becomes "small" in string units, in such a way that supergravity cannot be used to describe the system.

\section{Discussion}

We have shown in this note how some $D$-dimensional $A d S_{d+1} \times M_{D-d-1}$ spaces exhibit aspects of a holographic behaviour, also at the quantum level from the supergravity point of view. This was done by demonstrating that such systems give rise to a quantum entropy of massless states propagating in $d$ dimensions. This turned out to occur for the manifold $X_{2}$ and not for $X_{1}$. The presense of the infrared cut-off (from the point of view of the CFT on the boundary), imposed by the black hole in the supergravity master field, was responsible for that feature.

Probing the system at high tempreture is a rather crude way of testing neccesary conditions for holography. It would be useful if one could identify a-priori if a given string background obeys the necessary condition for holography. A possible way to encode such information would be in the value of the effective central charge of the theory, $c_{\text {eff }}$. If it would turn out correspond to the value $d$, it could be expected to be holographic. A familiar example is [17] the Liouville model. In the presence of a linear term for the Liouville field the central charge $c$ can increase indefinetely, but the value of $c_{\text {eff }}$ retains the value one, which is the value of $c$ in the absence of the linear term. If for example the manifold $X_{1}$ would have $c_{\text {eff }}=15$ while the manifold $X_{2}$ would have $c_{\text {eff }}=6$, then the holography properites would be more clearly manifested. For this to occur the presense of the black hole horizon should increase the weight of the ground-state appropriately. One may envisage even that backgrounds appropriate for low temperature for which $c=c_{\text {eff }}$

are dominated at high temperatures by manifolds for which $c$ is larger than $c_{\mathrm{eff}}$. This would endow holographic properties on the physics around general backgrounds. It may 
seem that in this case the value of the supergravity action allows one to choose among two different allowed string backgrounds, a criteria for such a choice is absent from usual string theory. One should recall however that [18] in gravitational systems it is $c_{\text {eff }}$ which is reduced by the renormalization group flow. Such a flow could represent the phase transition occuring in the supergravity picture in a string framework. It is however possible that the appropriate mechanism in string theory is some non-perturbative tunneling effect, of order $e^{-N^{2}}$.

A crucial ingredient of the holographic properties of Anti-de Sitter space is its nonextensivity at infinity. In other words, the thermal ensemble behaves like in a finite volume, the optical volume being actually finite. This means that the blown up theory at the throat is properly decoupled from the bulk gravity degrees of freedom at the asymptotically flat region of the brane solution, also to order $O\left(N^{0}\right) \sim O(1)$ in the large $N$ expansion. This is a rather non-trivial property which is violated in some examples which satisfy perturbative decoupling of gauge and gravity degrees of freedom.

Consider for example the non conformal Super Yang-Mills theories on the worldvolume of $N$ Dp-branes. The blown-up throat at large radius [19] can be convenientely cast in the form of an "adiabatic" product $A d S_{p+2} \times S^{8-p}$, with an $r$-dependent radius of curvature $b_{r}$ given by

$$
b_{r}^{2}=r^{\frac{p-3}{2}} b^{\frac{7-p}{2}}
$$

with $b \equiv \sqrt{\alpha^{\prime}}(\lambda N)^{\frac{1}{7-p}}$. The optical metric for this space at large $r$ is

$$
\widetilde{d s}^{2} \rightarrow\left(d x_{\|}\right)_{p+1}^{2}+\frac{b_{r}^{4}}{r^{4}} d r^{2}+\frac{b_{r}^{4}}{r^{2}} d \Omega_{8-p}^{2}
$$

The optical volume is finite for $p<5$, logarithmically divergent for $p=5$, and powerdivergent for $p>5$. The resulting free energy, calculated along the lines of (3.2) and (3.7), scales like a massless free energy in $p+1$ dimensions $\beta F \sim \beta^{-p}$, as long as $p<5$. However, it is dominated by ten-dimensional scaling $\beta F \sim \beta^{-9}$ for $p \geq 5$. This happens in spite of the fact that the asymptotic region up the throat is more smooth for larger $p$, and it means that gauge/gravity decoupling might be violated at large $g_{Y M}^{2} N$, even if it is satisfied for small 't Hooft coupling. Our result means that the breakdown of holography will be apparent at the level of $1 / N^{2}$ corrections [16]. 


\section{Acknowledgements}

We thank I. Klebanov and D. Kutasov for discussions. The work of E. R. is partially supported by the Israel Academy of Sciences and Humanities-Centers of Excellence Programme, and the American-Israel Bi-National Science Foundation. 


\section{References}

[1] G. 't Hooft, "Dimensional Reduction In Quantum Gravity", Salamfest 1993, p. 284, gr-qc/9310026;

J.D. Bekenstein, Phys. Rev. D49 (1994) 1912;

L. Susskind, J. Math. Phys. 36 (1995) 6377, hep-th/9409089.

[2] J. Maldacena, "The Large N Limit Of Superconformal Field Theories And Supergravity", hep-th/9711200.

[3] M. R. Douglas, J. Polchinksi, and A. Strominger, "Probing five dimensional black holes with D-branes", JHEP-12 (1997) 003 , hep-th/9703031.

[4] S.S. Gubser, I.R. Klebanov, and A. M. Polyakov, "Gauge Theory Correlators From Noncritical String Theory", hep-th/9802109.

[5] E. Witten, "Anti De Sitter Space and Holography", hep-th/9802150.

[6] S. S. Gubser, I. R. Klebanov and A. W. Peet, Phys. Rev. D54 (1996) 3915 hepth/9602135.;

I. R. Klebanov, Nucl. Phys. B496 (1997) 231 hep-th/9702076;

S. S. Gubser, I. R. Klebanov and A. Tseytlin, Nucl. Phys. B499 (1997) 217 hepth/9793940;

S. S. Gubser and I. R. Klebanov, Phys. Lett. B413 (1997) 41 hep-th/9708005.

[7] S. Hawking and D. Page, Commun. Math. Phys. 78B (1983) 577.

[8] L. Susskind and E. Witten, "The Holographic Bound in Anti-de Sitter Space", hepth/9805114.

[9] E. Witten, "Anti-de Sitter Space, Thermal Phase Transition, And Confinement In Gauge Theories", hep-th/9803131.

[10] G. W. Gibbons and S. Hawking, Phys. Rev. D15 (1977) 2752.

[11] G. W. Gibbons and M. J. Perry, Proc. R. Soc. Lond. A358, (1978), 467 ;

J.S. Dowker and G. Kennedy, J. Phys. A11 (1978) 895.

[12] G. 't Hooft, Nucl. Phys. B256 (1985) 727.

[13] L. Susskind and J. Uglum, Phys. Rev. D50 (1994) 2700.

[14] G. T. Horowitz and H. Ooguri, "Spectrum Of Large $N$ Gauge Theory From Supergravity", hep-th/9802116.

[15] T. Banks and M.B. Green, "Non-perturbative Effects in $A d S_{5} \times S^{5}$ String Theory and $d=4$ SUSY Yang-Mills", hep-th/9804170.

[16] J.L.F. Barbón and E. Rabinovici, to appear. 
[17] D. Kutasov and N. Seiberg, Nucl. Phys. B358 (1991) 600.

[18] D. Kutasov, Mod. Phys. Lett. A7 (1992) 2943;

E. Hsu and D. Kutasov, Nucl. Phys. B396 (1993) 693.

[19] N. Itzhaki, J. Maldacena, J. Sonnenschein, and S. Yankielowicz, "Supergravity And The Large N Limit Of Theories With Sixteen Supercharges", hep-th/9802042. 